\title{
ESTRUCTURA Y COMPOSICIÓN DE LA VEGETACIÓN DEL BOSQUE DE NIEBLA DE COPALILLOS, SAN LUIS POTOSÍ, MÉXICO
}

\author{
Javier Fortanelli-Martínez ${ }^{1,2}$, José García-Pérez ${ }^{1}$ y Pedro Castillo-Lara ${ }^{1}$ \\ ${ }^{1}$ Universidad Autónoma de San Luis Potosí, Instituto de \\ Investigación de Zonas Desérticas, Altair 200, Fracc. Del Llano, \\ 78377 San Luis Potosí, San Luis Potosí, México. \\ ${ }^{2}$ Autor para la correspondencia: fortanel@uaslp.mx
}

\section{RESUMEN}

Se presenta la lista florística y se describe la estructura de un bosque de niebla en Copalillos, San Luis Potosí, el cual, hasta hace muy poco tiempo estuvo ausente en la cartografía de vegetación existente. Se registraron 199 especies, correspondientes a 165 géneros y 91 familias botánicas. La estructura vertical mostró tres estratos arbóreos, uno arbustivo y otro herbáceo. Las especies dominantes en el arbóreo superior fueron Liquidambar styraciflua, Quercus germana y $Q$. pinnativenulosa. En el arbóreo intermedio destacaron Magnolia schiedeana, Persea liebmannii e Ilex rubra. El arbóreo inferior estuvo ampliamente dominado por Eugenia xalapensis. Este bosque de niebla relicto representa un eslabón importante dentro del conjunto de áreas de este tipo de vegetación situadas entre Hidalgo y Tamaulipas.

Palabras clave: bosque mesófilo de montaña, Sierra Madre Oriental.

\begin{abstract}
The floristics and structure of a cloud forest in Copalillos, San Luis Potosí, is presented. Until very recently, this forest was not recorded in the existing cartography. A total of 199 species, corresponding to 165 genera and 91 botanical families, were recorded. The forest structure consists of three arboreal layers (upper, middle and lower), besides shrub and herbaceous strata. The dominant species in the upper tree stratum are Liquidambar styraciflua, Quercus germana and Q. pinnativenulosa. The main tree species of the middle level were Magnolia schiedeana, Persea liebmannii and Ilex rubra. The lower tree layer was
\end{abstract}


largely dominated by Eugenia xalapensis. This relict represents an important link within the set of cloud forest areas located between Hidalgo and Tamaulipas.

Key words: cloud forest, Sierra Madre Oriental.

\section{INTRODUCCIÓN}

El bosque deciduo templado (Rzedowski, 1965), bosque mesófilo de montaña (Miranda 1947; Rzedowski, 1978), bosque de niebla (Williams, 2007), bosque caducifolio húmedo de montaña (Puig, 1991) o bosque húmedo de montaña (Villaseñor, 2010), ha sido ampliamente estudiado en México a partir de los trabajos de Miranda y Sharp (1950), Hernández et al. (1951) y Martin (1955) entre otros. La mayor parte de los autores coincide en que este tipo de vegetación es muy diverso, tiene una alta riqueza de especies de plantas vasculares (3000 a 5000) en relación con la escasa superficie que ocupa ( 0.8 a $1 \%$ del territorio nacional), que comparte elementos de floras de origen boreal y tropical, y que posee una estructura multiestratificada con abundantes epífitas y helechos. Estos atributos derivan del subsidio energético que implica el estar situado en zonas con procesos frecuentes de formación de niebla debidos principalmente al forzamiento del ascenso orográfico de los vientos húmedos procedentes de los cuerpos oceánicos.

Los bosques de niebla de México se distribuyen de forma archipelágica, en laderas pronunciadas (frecuentemente orientadas hacia barlovento) o en cañadas húmedas, al sur del paralelo $25^{\circ} \mathrm{N}$, en altitudes variables (desde 600 hasta $3200 \mathrm{~m}$ ) (González et al., 2011). Este tipo de vegetación se presenta en mayores extensiones en la Sierra Madre Oriental, la Sierra Norte de Oaxaca, la Sierra Madre del Sur, las montañas del norte de Chiapas y la Sierra Madre de Chiapas (González et al., 2011). Los bosques de niebla de San Luis Potosí han sido estudiados muy marginalmente; Rzedowski (1965) y Puig (1991) ubican como área destacada por este tipo de cobertura a Xilitla. Además del municipio anterior, Rzedowski (1965) menciona a Tamazunchale y Aquismón, en tanto que Puig (1991) añade a Ciudad del Maíz, Cárdenas y Rioverde. Sin embargo, el tratamiento que se da a los vestigios existentes en la porción septentrional de la Sierra Madre Oriental potosina es el de encinares mezclados con liquidámbar (Rzedowski, 1965). Ésta también fue la apreciación de Martin (1955), para quien no existen verdaderos bosques húmedos de montaña entre Xilitla y El Cielo (en Tamaulipas), y solo refiere como algo aproximado, un bosque de Quercus, Liquidambar y Cercis al este del poblado de Ciudad del Maíz (Davis y Johnston 1947, citados por Martin, 1955). La cartografía de la Comisión de Estudios del Territorio Nacional (Anónimo, 1973) mues- 
tra varias zonas situadas en Alaquines y Ciudad del Maíz también como asociaciones de Quercus y Liquidambar. Otros autores (Foroughbakhch et al., 2004) refieren la existencia de un bosque de Liquidambar styraciflua, Quercus canbyi y Q. rysophylla, entre Ciudad del Maíz y El Naranjo (localidad El Platanito). Más recientemente, la Comisión Nacional para el Conocimiento y Uso de la Biodiversidad (CONABIO) señala a estos rodales claramente con la categoría de bosque mesófilo de montaña y los localiza al sur de la Sierra Madre Oriental Plegada (Subregión Vestigios de San Luis Potosí), en los municipios de Alaquines, Ciudad del Maíz y El Naranjo (Anónimo, 2010a). En esta misma obra se ubica a los correspondientes de los alrededores de Xilitla en la región Huasteca Alta Hidalguense. Una cartografía más detallada fue realizada por Leija et al. (2011), quienes refieren la existencia de ocho áreas de bosque de niebla distribuidos en los municipios de Xilitla, Rayón, Tamasopo, Alaquines, El Naranjo y Ciudad del Maíz; su extensión suma 3239 ha.

De acuerdo con la Comisión Nacional para el Conocimiento y Uso de la Biodiversidad (Anónimo, 2010a), es indispensable realizar investigación biológica y ecológica en la Subregión Vestigios de San Luis Potosí. Al respecto se ha estado trabajando en esta subregión desde 2004, y algunos resultados se han dado a conocer de forma sucinta en congresos botánicos (García y Fortanelli, 2005, 2010). Sin embargo, aún no se han publicado de manera formal y detallada. Por esta razón, el objetivo del presente trabajo es caracterizar estructural y florísticamente el vestigio de bosque de niebla de Copalillos, situado en los municipios de Rayón y Tamasopo. Este lugar no aparece como tal (sino con otras denominaciones no equivalentes) en la cartografía del Instituto Nacional de Estadística y Geografía y de la Comisión Nacional para el Conocimiento y Uso de la Biodiversidad, y no ha sido mapeado como bosque de niebla antes del trabajo de Leija et al. (2011). La importancia del mismo reside en que es el primer vestigio localizado aproximadamente a $58 \mathrm{~km}$ al noroeste de Xilitla, después de una interrupción causada por el descenso orográfico hacia el río Santa María, y su descripción podría aportar datos de interés que contribuyan a la formulación de hipótesis de trabajo para el estudio biogeográfico del espacio situado entre Xilitla, San Luis Potosí y El Cielo, Tamaulipas.

\section{MATERIALES Y MÉTODOS}

Área de estudio

De acuerdo con Leija et al. (2011), el bosque de niebla de Copalillos tiene una extensión de 147.4 ha (en 1973 contaba con 266.8 ha). Se encuentra ubicado en los 
municipios de Tamasopo y Rayón, entre las coordenadas $2414300 \mathrm{~N}$ a $2417681 \mathrm{~N}$, y 453074E a 454006E, Zona 14Q (Fig. 1), equivalentes a $21^{\circ} 49^{\prime} 55^{\prime \prime}-21^{\circ} 51^{\prime} 45^{\prime \prime} \mathrm{N}$; 99 $26^{\prime} 43^{\prime \prime}-99^{\circ} 27^{\prime} 15^{\prime \prime} \mathrm{O}$, que abarcan solo las áreas donde aún se observan remanentes de bosque de niebla con predominio de liquidámbar. Las altitudes extremas son 1075 y $1325 \mathrm{~m}$. El material geológico consiste principalmente de lutitas, o de estratos intercalados de lutitas y calizas. En sus flancos oriental y occidental afloran rocas calizas. Las geoformas locales son pequeñas sierras que se alinean en dirección de los principales plegamientos (NW-SE), separadas por algunas cañadas. El sistema hidrológico superficial lo constituyen los arroyos Hondo, El Carrizal y Agua Zarca, los cuales se originan de manantiales, son de corto trayecto y se pierden en resumideros. El vestigio se encuentra en la zona limítrofe entre los climas semicálido húmedo con abundantes lluvias en verano y semicálido subhúmedo (Anónimo, 2002a). Se carece de datos precisos de precipitación media anual para la zona, aunque la estación meteorológica más cercana (Agua Buena) registra arriba de $1800 \mathrm{~mm}$. Carbajal (2008), mediante una técnica de interpolación estimó $1350 \mathrm{~mm}$ para la cercana comunidad Las Guapas en el municipio de Rayón; lo anterior hace suponer que la precipitación media anual para la zona de estudio debe tener un valor situado entre los 1300 y los $1500 \mathrm{~mm}$. Los suelos corresponden a litosoles en las laderas y a regosoles en las zonas donde el horizonte A alcanza mayor espesor; en el fondo de las dolinas cársticas se presentan rendzinas. El uso del suelo, aparte del aprovechamiento del bosque, se divide en agrícola y pecuario; en el primer caso se cultiva maíz (Zea mays), asociado con frijol (Phaseolus vulgaris, P. coccineus) y calabaza (Cucurbita spp.); en las áreas ganaderas se suele establecer zacate estrella de África (Cynodon plectostachyum) o bien propiciar la presencia de otras gramíneas como Setaria parviflora, Sporobolus indicus, etc. La calidad de los sitios de apacentamiento es variable, aunque en algunos lugares es evidente el sobrepastoreo por la presencia de plantas arbustivas invasoras como Acacia farnesiana, Vernonia patens y Baccharis conferta, entre otras.

\section{Trabajo de campo}

Se verificó en el campo la información cartográfica sobre topografía, litología superficial, hidrología superficial, suelos y vegetación y se indagó sobre las diferentes formas de uso del suelo. Se efectuaron recorridos con guías conocedores de los senderos para realizar muestreos botánicos. Se recolectaron los especímenes que tenían los caracteres morfológicos necesarios para su identificación taxonómica. Se registró el nombre local, la ubicación del sitio, sus características físicas y bióticas y la forma vital de la especie. Los ejemplares se curaron, identificaron y depositaron en el herbario Isidro Palacios (SLPM). En aquellos casos en los que la asignación de la especie era imprecisa se acudió a espe- 
Fortanelli-Martínez et al.: Estructura y composición del bosque mesófilo de Copalillos, México

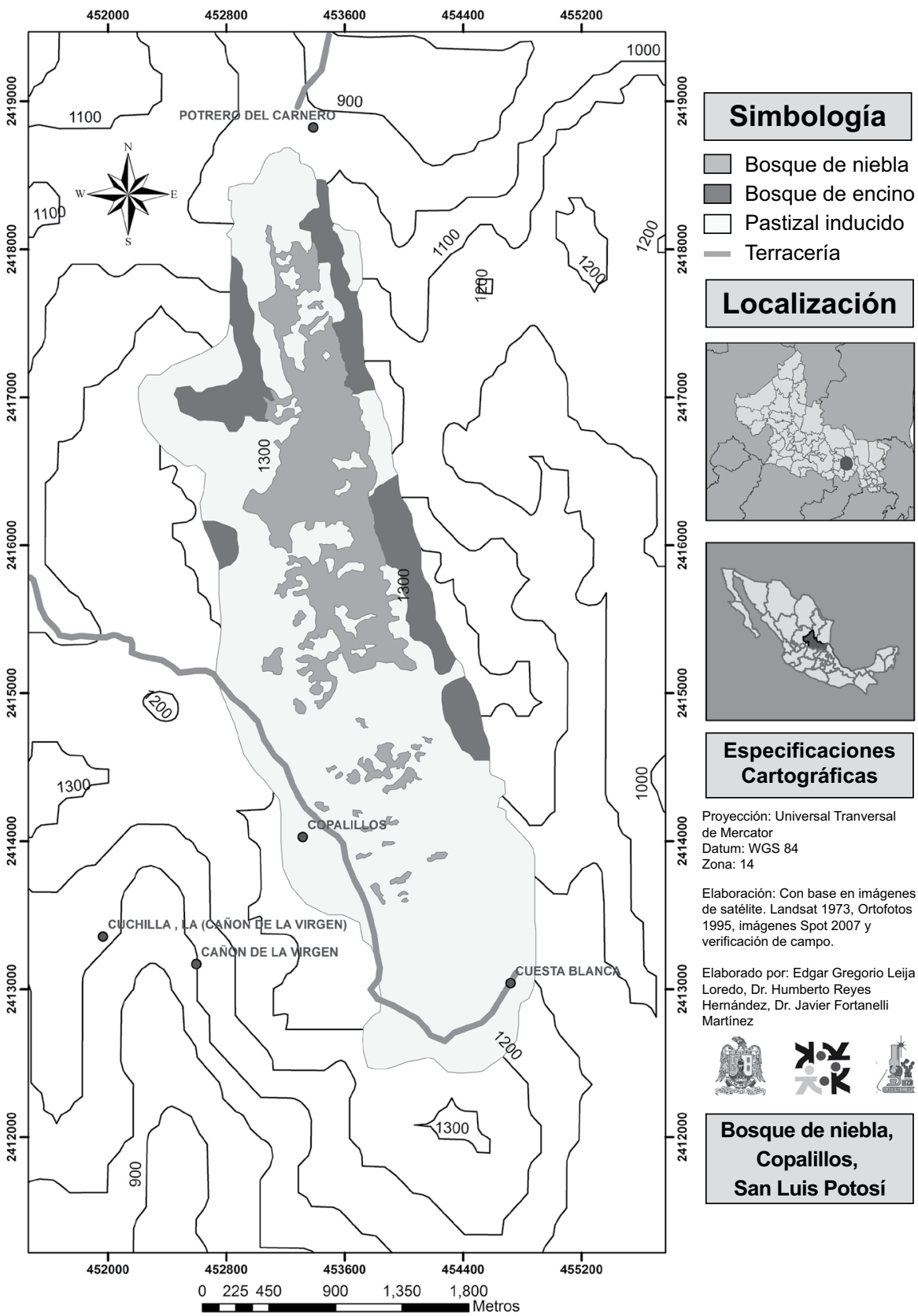

Fig. 1. Localización del vestigio de bosque de niebla de Copalillos, San Luis Potosí (Autor: Édgar Gregorio Leija Loredo, adaptado de Leija, 2010). 
cialistas de la Facultad de Ciencias y del Instituto de Biología de la Universidad Nacional Autónoma de México, del Instituto Politécnico Nacional, de la Universidad Autónoma de Querétaro y del Instituto de Ecología A. C. (sede Xalapa y Centro Regional del Bajío en Pátzcuaro).

Se consideró importante para los fines de este trabajo efectuar una comparación entre las especies arbóreas del bosque de niebla de Copalillos y las registradas por Luna et al. (1994) para Tlanchinol, Cartujano et al. (2002) para Landa de Matamoros, y Puig et al. (1987) para El Cielo. Para hacer compatibles los datos se actualizó la nomenclatura de muchos de los elementos y se calculó el coeficiente de comunidades (también llamado índice de similitud) de Sorensen (Smith y Smith, 2002). Para evaluar la estructura de la vegetación se eligieron cuatro unidades de estudio, por su buen estado de conservación, con dimensiones de $50 \mathrm{~m}$ x $50 \mathrm{~m}\left(2500 \mathrm{~m}^{2}\right)$. Cada unidad se dividió en 25 subunidades de $100 \mathrm{~m}^{2}$, de las cuales aleatoriamente se eligieron cinco para realizar el muestreo de vegetación. En cada subunidad se midieron el diámetro del tallo (a $1.30 \mathrm{~m}$ de altura a partir de la base (DAP) de los todos los individuos $\geq 2.5$ $\mathrm{cm}$ de diámetro), y la altura de las especies arbóreas. Para su análisis, estas últimas se agruparon en tres estratos de acuerdo con su altura promedio. Dichos niveles fueron: a) superior, con individuos $\geq 20 \mathrm{~m}$ de altura; b) medio, entre 10 y $20 \mathrm{~m}$ de altura; y c) inferior, $<10 \mathrm{~m}$ de altura. De los elementos del estrato arbustivo se midieron el diámetro basal y la altura. Se registró el nombre común y/o el nombre científico de cada uno de los individuos encontrados. De acuerdo con su forma de crecimiento (Moreno, 1984), se consideró como árboles a todos aquellos individuos perennes, con un tallo lignificado (el cual se ramifica por arriba de la base) y que, generalmente, miden más de 3 $\mathrm{m}$ de altura; mientras que los arbustos son aquellos organismos vegetales perennes, con tallo lignificado (el cual se ramifica desde la base), usualmente menores de $3 \mathrm{~m}$ de altura. Del estrato herbáceo se estimó la frecuencia y se midió la altura de las especies observadas. En el caso de las epífitas y trepadoras, únicamente se registró su presencia y la planta arbórea o arbustiva sobre la que crecían. Con los datos obtenidos se determinaron la densidad, cobertura y frecuencia, así como el valor de importancia relativo para los elementos de los estratos arbóreo y arbustivo. Dichos valores se calcularon de acuerdo con Mueller-Dombois y Ellenberg (1974) y Stiling (1999).

\section{RESULTADOS Y DISCUSIÓN}

\section{Florística}

En el Apéndice 1 se presenta la lista florística correspondiente a este bosque de niebla. En total, se registraron 199 especies de plantas vasculares, pertenecientes 
a 165 géneros y 91 familias. Dentro de las últimas destacan Asteraceae (22 especies), Fabaceae (16), Rubiaceae (9), Fagaceae (8), Solanaceae (6), Smilacaceae (5), Malvaceae (5) y Orchidaceae (5). Se encontraron 41 árboles, 38 arbustos, 84 hierbas, 10 trepadoras leñosas, 18 trepadoras no leñosas, 5 epífitas, 1 parásita y 1 hemiparásita.

Es importante destacar que las principales especies arbóreas, como Liquidambar styraciflua, Quercus germana, $Q$. pinnativenulosa y $Q$. xalapensis se distribuyen ampliamente en este bosque; sin embargo, otras que corresponden a estratos de menor porte, como Magnolia schiedeana, Clethra pringlei, Carya ovata var. mexicana, Persea liebmannii, y Quercus rysophylla se presentan de manera discontinua en rodales con variadas condiciones ambientales o con diferente antigüedad. Lo mismo ocurre con aquellas que ocupan el estrato arbóreo inferior, como Chione venosa subsp. mexicana, Styrax glabrescens, Ostrya virginiana, Sambucus canadensis o Saurauia aspera.

Los gradientes altitudinales también parecen influir sobre la distribución de algunas especies; por ejemplo, Dendropanax arboreus se presenta solo por debajo de los 1100 m s.n.m., situación semejante a la de Sapindus saponaria. Otro factor evidentemente significativo es la litología superficial; en especial, resalta la afinidad de Liquidambar styraciflua y Carya ovata var. mexicana por las lutitas, ya que no se observan sobre calizas; en contraste, Juglans mollis, Quercus laeta y Q. polymorpha solo crecen sobre sustrato calizo.

La especie más importante en el nivel del sotobosque es Eugenia xalapensis, la cual forma poblaciones densas, por lo general en condiciones umbrías con alta presencia de musgos; este árbol suele servir de soporte a plantas trepadoras, principalmente de la familia Smilacaceae. Los helechos usualmente se encuentran en ambientes más húmedos como las exposiciones norte y noreste así como en los márgenes de arroyos; se encontró Alsophila firma, un helecho de tipo arborescente, así como otros propios del estrato arbustivo como Marattia weinmanniifolia, Ctenitis melanosticta, Dennstaedtia distenta, Diplazium franconis y Lophosoria quadripinnata. Elementos adicionales que suelen verse en el estrato arbustivo son Deppea purpusii, Leandra cornoides, Randia laetevirens y Xylosma flexuosa. En algunas áreas húmedas y umbrías se observa Heliconia schiedeana, Piper auritum, Sambucus canadensis y Urera caracasana. En el estrato herbáceo destacan Achimenes grandiflora, Arisaema macrospathum, Aulosepalum ramentaceum, Begonia incarnata, Botrychium schaffneri, Ceratozamia mexicana var. latifolia, Iresine diffusa, Ocimum selloi, Phytolacca icosandra, $P$. rivinoides, $P i$ lea pubescens, Ponthieva racemosa, Piper umbellatum y Tibouchina galeottiana. Las trepadoras constituyen un componente importante; sobresalen Smilax bona- 
nox, S. glauca, S. mollis, S. moranensis y S. pringlei. Además de las anteriores se presentan Bomarea hirtella, Gonolobus niger, Marsdenia pringlei, Metastelma angustifolium, Perymenium ovalifolium, Syngonium podophyllum, Toxicodendron radicans y Vitis berlandieri. Dentro de las epífitas destaca Tillandsia deppeana; con menor relevancia aparecen Campyloneuron angustifolium, Echeveria rosea, Isochillus unilateralis y Tillandsia bartramii. En las áreas con disturbio suelen observarse Acmella repens, Ageratina petiolaris, Asclepias curassavica, Bouvardia laevis, Brugmansia candida, Calea urticifolia, Cestrum oblongifolium, Cirsium mexicanum, Cnidoscolus multilobus, Desmodium glutinosum, D. prehensile, Fleischmannia pycnocephala, Ipomoea purpurea, Oenothera rosea, Pennisetum clandestinum, Plantago lanceolata, Prunus persica, Psidium guajava, Roldana aschenborniana, R. lanicaulis, Rubus adenotrichos, Sclerocarpus uniserialis, Solanum myriacanthum, S. seaforthianum, S. torvum, Tagetes lucida, T. filifolia, Tigridia pavonia y Zingiber officinale.

El número de especies encontrado se puede comparar con los 150 elementos (con 60\% determinado hasta el nivel de especie) referidos por Puig et al. (1987) para El Cielo, Tamaulipas, en un área de $100 \mathrm{~km}^{2}$ y una amplitud altitudinal entre los 800 y los $1500 \mathrm{~m}$. Estos autores mencionan como las familias más importantes a Fagaceae, Rubiaceae y Solanaceae, mismas que aparecen en Copalillos en cuarto, quinto y sexto lugar, respectivamente. Por su parte, Luna et al. (1994) encontraron en Tlanchinol, Hidalgo, 336 especies, 247 géneros y 107 familias; en este caso se trata de un área considerablemente mayor que la del bosque de Copalillos (probablemente 15 veces más grande) y con un espacio altitudinal más amplio, entre los 900 y los 1800 $\mathrm{m}$ (el intervalo altitudinal del bosque de Copalillos va de los 1075 a los $1325 \mathrm{~m}$ ). Un número aún mayor de especies, géneros y familias (774, 465 y 130, respectivamente) fue encontrado por Cartujano et al. (2002) en varios fragmentos de bosque mesófilo de montaña del municipio de Landa de Matamoros, Querétaro. En este caso, los rodales estudiados se localizan en elevaciones entre los 600 y los 2100 m s.n.m.

En el Apéndice 2 se presenta la relación de los elementos arbóreos del bosque de niebla registrado en Tlanchinol por Luna et al. (1994), en El Cielo por Puig et al. (1987), en Landa de Matamoros por Cartujano et al. (2002) y lo encontrado en el presente estudio. Salvo posibles errores por ubicación de las especies en estratos diferentes o por identificación incorrecta, las mayores coincidencias se dan con El Cielo (Coeficiente de comunidades de Sorensen $=0.4691 ; 41$ especies en Copalillos, 40 especies en El Cielo y 19 especies compartidas), las cuales podrían incrementarse con una revisión de los géneros Ilex, Prunus, Zanthoxylum, Bernardia y Eugenia para precisar su identidad taxonómica. Un valor semejante se obtiene en la compa- 
ración entre Copalillos y Landa (0.4340; 41 especies en Copalillos, 65 en Landa y 23 especies compartidas). En contraste, el coeficiente de comunidades del estrato arbóreo entre Tlanchinol y Copalillos es apenas de 0.2095 (41 especies en Copalillos, 64 especies en Tlanchinol, 11 especies compartidas). La semejanza entre El Cielo y Tlanchinol, de acuerdo con tal coeficiente, es de 0.1731, y entre Landa y Tlanchinol es de 0.2326 . Estos resultados deben analizarse con cautela, dado que corresponden a unidades de muestreo con extensiones, amplitudes altitudinales y grados de disturbio diferentes. A reserva de lo anterior, los datos sugieren que el bosque de Copalillos se asemeja más a los bosques de Landa y El Cielo que al de Tlanchinol. En lo anterior probablemente influya el gradiente latitudinalmente decreciente de humedad (S-N). Es importante destacar que la barrera que supondrían las sierras de baja altitud aledañas al río Santa María no influye de manera importante en la diferenciación de la flora arbórea de Copalillos y Landa; asimismo, se presenta una menor semejanza de ambas con Tlanchinol, situada más al sur. Sin embargo, la flora arbórea de Copalillos se enlaza con la de los otros bosques por la presencia de $\mathrm{Li}$ quidambar styraciflua, Quercus germana, Q. xalapensis, Myrsine coriacea subsp. coriacea y Ostrya virginiana. Las cuatro primeras especies son propias de las áreas montañosas de la vertiente del Golfo de México hasta alcanzar Centroamérica. Ostrya virginiana se distribuye sobre las cadenas montañosas del este de México y del Pacífico, y se extiende desde Honduras hasta el límite noreste entre Estados Unidos y Canadá. Resulta también interesante visualizar a Copalillos como un área de enlace con el sur de la Sierra Madre Oriental, como lo muestran Styrax glabrescens, Myrsine coriacea, Eugenia xalapensis y Chione venosa subsp. mexicana. En general, gran parte de las especies compartidas con el bosque de El Cielo tienen amplia distribución sobre la mencionada cadena montañosa. Algunas que usualmente se presentan en elevaciones mayores a los 1300 m s.n.m., como Pinus patula, P. greggii, Taxus globosa o Podocarpus reichei no se encuentran en Copalillos por carecer este sitio de tales pisos altitudinales.

Estructura de la vegetación

Las especies arbóreas se pueden agrupar, de acuerdo con su altura, en tres estratos: a) superior (árboles de 20 a más de $30 \mathrm{~m}$ de altura), b) medio (de entre 10 y $20 \mathrm{~m}$ de altura), y c) inferior, arbolillos menores de $10 \mathrm{~m}$ de altura. En el arbóreo superior, Liquidambar styraciflua es la especie dominante, seguida por Quercus pinnativenulosa y $Q$. germana (Cuadro 1). En el arbóreo intermedio, la del mayor valor de importancia es Persea liebmannii, seguida por Magnolia schiedeana e Ilex rubra. El inferior está totalmente dominado por Eugenia xalapensis. 
Cuadro 1. Parámetros estructurales del estrato arbóreo del bosque de niebla de Copalillos, S.L.P.

\begin{tabular}{|c|c|c|c|c|c|}
\hline Especie & $\begin{array}{l}\text { Altura } \\
\text { promedio } \\
(\mathrm{m})\end{array}$ & Densidad* & $\begin{array}{l}\text { Área basal } \\
(\mathrm{m} 2)^{*}\end{array}$ & Frecuencia & $\operatorname{IVIR}^{* *}$ \\
\hline \multicolumn{6}{|c|}{ Estrato arbóreo superior (>20 m) } \\
\hline Liquidambar styraciflua & 27.37 & 250 & 23.16 & 1.0 & 47.27 \\
\hline Quercus pinnativenulosa & 23.78 & 53 & 15.49 & 1.0 & 31.93 \\
\hline Quercus germana & 24.29 & 120 & 10.04 & 1.0 & 25.49 \\
\hline Clethra pringlei & 21.08 & 53 & 3.93 & 1.0 & 15.09 \\
\hline Carya ovata var. mexicana & 20.75 & 26 & 1.47 & 0.6 & 8.13 \\
\hline \multicolumn{6}{|c|}{ Estrato arbóreo medio $(10-20 \mathrm{~m})$} \\
\hline Persea liebmannii & 19.35 & 73 & 2.77 & 0.6 & 11.11 \\
\hline Ilex rubra & 13.29 & 66 & 0.99 & 0.6 & 8.39 \\
\hline Magnolia schiedeana & 13.31 & 107 & 2.15 & 0.3 & 8.33 \\
\hline Ostrya virginiana & 12.84 & 13 & 0.21 & 0.6 & 6.00 \\
\hline Bernardia dodecandra & 15.00 & 33 & 0.05 & 0.3 & 3.55 \\
\hline Zanthoxylum clava-herculis & 12.36 & 7 & 0.05 & 0.3 & 2.93 \\
\hline \multicolumn{6}{|c|}{ Estrato arbóreo inferior $(<\mathbf{1 0} \mathrm{m})$} \\
\hline Eugenia xalapensis & 2.91 & 3166 & 6.91 & 1.0 & 93.23 \\
\hline Styrax glabrescens & 7.53 & 107 & 0.59 & 0.3 & 6.07 \\
\hline Prunus serotina & 6.38 & 6 & 0.76 & 0.3 & 3.94 \\
\hline Morus celtidifolia & 6.00 & 27 & 0.35 & 0.3 & 3.83 \\
\hline Cinnamomum effusum & 5.80 & 33 & 0.09 & 0.3 & 3.62 \\
\hline Cnidoscolus multilobus & 1.50 & 26 & 0.02 & 0.3 & 3.35 \\
\hline $\begin{array}{l}\text { Myrsine coriacea subsp. } \\
\text { coriacea }\end{array}$ & 7.25 & 7 & 0.30 & 0.3 & 3.28 \\
\hline Cercis canadensis & 8.35 & 7 & 0.09 & 0.3 & 2.98 \\
\hline Quercus xalapensis & 7.00 & 7 & 0.03 & 0.3 & 2.89 \\
\hline Crataegus mexicana & 5.64 & 7 & 0.02 & 0.3 & 2.88 \\
\hline Trema micrantha & 7.64 & 7 & 0.01 & 0.3 & 2.86 \\
\hline Daphnopsis mollis & 1.64 & 6 & 0.01 & 0.3 & 2.85 \\
\hline
\end{tabular}

* Promedio por hectárea.

** IVIR: Índice de Valor de Importancia Relativo. Las especies se ordenaron en sentido descendente de acuerdo con este índice. 
En este bosque el estrato arbustivo, en general, no sobrepasó el metro de altura; las especies con los mayores valores de importancia son Deppea purpusii, Hoffmannia excelsa y Cestrum oblongifolium (Cuadro 2).

El estrato herbáceo es muy pobre, posiblemente debido a la intercepción de la luz por el dosel de árboles y arbustos. De esta forma solo algunas especies umbrófilas pueden desarrollarse en el mismo. Algunas de las más frecuentes son Pteris orizabae, Blechnum occidentale, Asplenium monanthes, Pecluma plumula y Begonia incarnata. Entre los bejucos y epífitas, las especies más frecuentes son Tillandsia deppeana y Toxicodendron radicans sobre Liquidambar styraciflua, y Smilax mollis, S. moranensis y $S$. glauca sobre Eugenia xalapensis.

Como puede apreciarse, el bosque de niebla de Copalillos se compone de cinco estratos bien definidos (tres estratos arbóreos, el arbustivo y el herbáceo) y presenta trepadoras leñosas, trepadoras no leñosas y epífitas. Los valores de densidad total (4099 individuos/ha) del estrato arbóreo superan ampliamente a los reportados por Puig et al. (1987) para el bosque de niebla de Tamaulipas, quienes registraron 1169 individuos/ha. En este caso, Puig et al. (1987) consideran como especies propias del estrato arbóreo a todos aquellos individuos leñosos con diámetros mayores de $5 \mathrm{~cm}$ a la altura del pecho. Asimismo, los datos de Copalillos son superiores a los publicados por Williams (2007) para el bosque de niebla del centro de Veracruz, quien registró 1035 individuos/ha. Williams (2007) no menciona los criterios de inclusión y únicamente señala que registró individuos arbóreos de más de cinco centímetros de diámetro a la altura del pecho. Aunque la densidad total mencionada por Williams (2007) es también muy inferior a la encontrada en Copalillos, se debe considerar que en ese estudio Eugenia xalapensis se incluyó en el estrato arbustivo, mientras que en el de Copalillos esta especie se asignó al nivel arbóreo inferior. Por lo tanto, cabría esperar solo una ligera superioridad de los datos de densidad de Copalillos. Con respecto al área basal, los registros obtenidos en Copalillos $\left(57 \mathrm{~m}^{2} / \mathrm{ha}\right)$ son muy semejantes a los datos publicados para Veracruz ( $58 \mathrm{~m}^{2} / \mathrm{ha}$; Williams, 2007) y ligeramente superiores a los reportados para Tamaulipas (31 $\mathrm{m}^{2} /$ ha; Puig et al., 1987). De las ocho especies con los mayores valores de área basal $\mathrm{en}^{2} /$ ha de El Cielo Tamaulipas, hay cinco compartidas con su equivalente de Copalillos: Liquidambar styraciflua, Quercus germana, Eugenia xalapensis, Magnolia schiedeana y Clethra pringlei. En relación con el atributo de densidad, nuevamente se presenta una coincidencia entre ambas localidades, pues la especie más importante en El Cielo es Eugenia capuli, mientras que en Copalillos este lugar lo ocupa Eugenia xalapensis (quizá una revisión cuidadosa de los ejemplares apunte a que se trata realmente de la mis- 
Cuadro 2. Parámetros estructurales del estrato arbustivo del bosque de niebla de Copalillos, S.L.P.

\begin{tabular}{lcrrrr}
\hline Especie & $\begin{array}{c}\text { Altura } \\
\text { promedio } \\
(\mathrm{m})\end{array}$ & $\begin{array}{r}\text { Densidad* } \\
\text { Área basal } \\
(\mathrm{m} 2)^{*}\end{array}$ & Frecuencia & IVIR** \\
\hline Deppea purpusii & 0.57 & 3240 & 1.17 & 1.00 & 103.07 \\
Marattia weinmanniifolia & 2.83 & 60 & 1.26 & 0.33 & 39.55 \\
Hoffmannia aff. excelsa & 0.33 & 980 & 0.25 & 0.66 & 34.14 \\
Ceratozamia mexicana & 0.56 & 280 & 0.29 & 1.00 & 28.46 \\
$\quad$ var. latifolia & & & & & \\
Dennstaedtia distenta & 0.75 & 120 & 0.60 & 0.33 & 23.18 \\
Randia laetevirens & 0.70 & 260 & 0.02 & 1.00 & 20.99 \\
Cestrum oblongifolium & 0.64 & 360 & 0.07 & 0.33 & 13.36 \\
Piper auritum & 0.18 & 260 & 0.02 & 0.33 & 10.30 \\
Senna septemtrionalis & 0.78 & 120 & 0.03 & 0.33 & 8.14 \\
Berberis gracilis & 0.68 & 20 & 0.05 & 0.33 & 6.93 \\
Heliconia schiedeana & 0.83 & 20 & 0.02 & 0.33 & 6.14 \\
Xylosma flexuosa & 0.46 & 40 & 0.00 & 0.33 & 5.74 \\
\hline
\end{tabular}

* Promedio por hectárea

** IVIR: Índice de Valor de Importancia Relativo. Las especies se ordenaron en sentido descendente de acuerdo con este índice.

ma especie) seguidas, en ambos casos por Liquidambar styraciflua. En términos generales, estas evidencias apoyan la idea de que existe una fuerte semejanza en el estrato arbóreo entre Copalillos y El Cielo, y que las diferencias se deben más bien a la mayor amplitud altitudinal del segundo bosque. Las especies con mayor valor de importancia relativo en Copalillos (Cuadro 1) fueron Eugenia xalapensis, Liquidambar styraciflua, Quercus pinnativenulosa, Q. germana y Clethra pringlei; sin embargo, se debe considerar que Eugenia xalapensis, un arbolillo propio del sotobosque, posee la más alta densidad, atributo que influyó decisivamente en el valor de importancia, tanto absoluto como relativo (Fig. 2). Si la comparación se hace solo sobre la base del estrato arbóreo superior las coincidencias con Tamaulipas claramente se incrementan.

Los valores relacionados con los niveles arbustivo y herbáceo no se comparan con los otros dos trabajos (El Cielo y Tlanchinol), debido a que ahí no fueron 
Fortanelli-Martínez et al.: Estructura y composición del bosque mesófilo de Copalillos, México

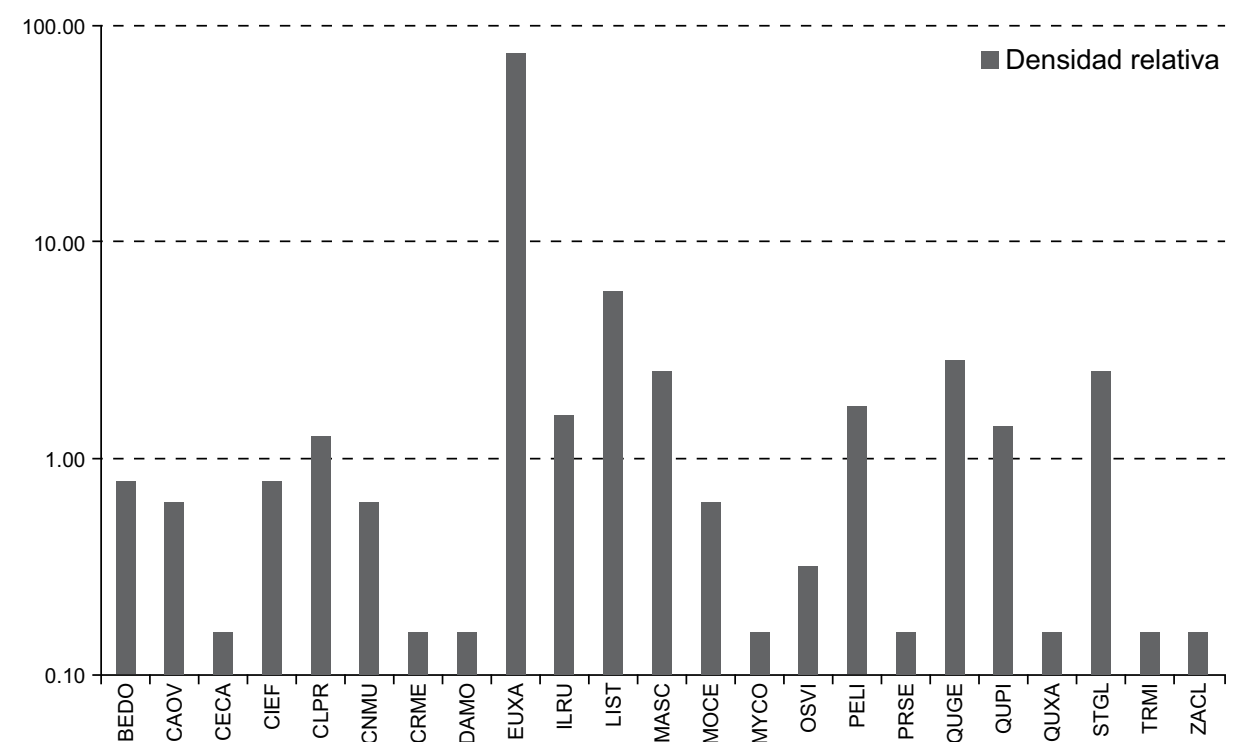

Fig. 2. Contribución de las especies del estrato arbóreo al valor de densidad relativa (BEDO $=$ Bernardia dodecandra $; \mathrm{CAOV}=$ Carya ovata var. mexicana $; \mathrm{CECA}=$ Cercis canadensis; $\mathrm{CIEF}=$ Cinnamomum effusum $; \mathrm{CLPR}=$ Clethra pringlei; $\mathrm{CNMU}=$ Cnidoscolus multilobus; $\mathrm{CRME}=$ Crataegus mexicana $; \mathrm{DAMO}=$ Daphnopsis mollis; EUXA = Eugenia xalapensis; ILRU = Ilex rubra; LIST = Liquidambar styraciflua; MASC = Magnolia schiedeana; MOCE = Morus celtidifolia $;$ MYCO = Myrsine coriace subsp. coriacea OSVI = Ostrya virginiana $;$ PELI = Persea liebmannii; PRSE = Prunus serotina $;$ QUGE = Quercus germana $;$ QUPI = Quercus pinnativenulosa; QUXA = Quercus xalapensis; STGL = Styrax glabrescens; TRMI = Trema micrantha; ZACL = Zanthoxylum clava-herculis).

cuantificados. Solamente en El Cielo se calcularon datos de abundancia y frecuencia para estos estratos, en los cuales Hoffmannia sp. y Psychotria erythrocarpa fueron las más abundantes en el estrato arbustivo, en tanto que Oplismenus hirtellus ssp. setarius y Tripogandra sp., lo fueron para el herbáceo.

\section{CONCLUSIONES}

En el bosque de niebla de Copalillos, San Luis Potosí, se registraron 199 especies, correspondientes a 165 géneros y 91 familias botánicas, principalmente Asteraceae (22 especies), Fabaceae (16), Rubiaceae (9), Fagaceae (8), Solanaceae (6), Smilacaceae (5), Malvaceae (5) y Orchidaceae (5). 
Se observó una estructura representada por tres estratos arbóreos, uno arbustivo y uno herbáceo, así como la presencia de trepadoras herbáceas y leñosas, además de epífitas.

En el estrato arbóreo superior las especies dominantes son Liquidambar styraciflua, Quercus pinnativenulosa y Q. germana. En el estrato arbóreo intermedio destacan Persea liebmannii, Magnolia schiedeana e Ilex rubra. El estrato arbóreo inferior está totalmente dominado por Eugenia xalapensis, la especie más ampliamente distribuida en este bosque.

Con base en el análisis de los datos florísticos y estructurales, el bosque de niebla relicto de Copalillos, San Luis Potosí, representa un eslabón importante dentro del conjunto de vestigios situados entre Hidalgo y Tamaulipas. De acuerdo con los datos obtenidos, su mayor semejanza con el de El Cielo refuerza la regionalización sugerida por la Comisión Nacional para el Conocimiento y Uso de la Biodiversidad (Anónimo, 2010a). Por ello se sugiere incluir esta localidad dentro del conjunto de bosques de niebla situados dentro de la subregión Vestigios de San Luis Potosí. Asimismo, la semejanza de los estratos arbóreos de Landa, Copalillos y El Cielo sugiere que esos bosques, podrían formar parte de una misma subregión.

Se recomienda establecer acciones de conservación en este bosque de niebla relicto, ya que en él se encuentran especies señaladas en la NOM-059-SEMARNAT-2010 (Anónimo, 2010b) en alguna de las categorías de riesgo, tales como Ostrya virginiana, Alsophila firma, Marattia weinmanniifolia, Magnolia schiedeana, Ceratozamia mexicana var. latifolia y Diospyros riojae; las tres primeras están enlistadas en la NOM-059 bajo el régimen de protección especial, Magnolia schiedeana está amenazada, y Ceratozamia mexicana var. latifolia y Diospyros riojae son especies en peligro de extinción.

\section{AGRADECIMIENTOS}

Se agradece a la Fundación Produce de San Luis, A.C. el apoyo otorgado para la realización del proyecto "Manejo y conservación de los relictos de bosque de niebla del ejido La Palma, Tamasopo, S.L.P.”, del cual forma parte esta investigación.

\section{LITERATURA CITADA}

Anónimo. 1973. Carta Uso del Suelo F-14-A-78. Comisión de Estudios del Territorio Nacional. México, D.F., México. 
Anónimo. 2002. Síntesis de información geográfica del estado de San Luis Potosí. Instituto Nacional de Estadística, Geografía e Informática. Aguascalientes, México. 112 pp. (anexo cartográfico).

Anónimo. 2010a. El bosque mesófilo de montaña en México. Amenazas y oportunidades para su conservación y manejo sostenible. Comisión Nacional para el Conocimiento y Uso de la Biodiversidad. México, D.F., México. 197 pp.

Anónimo. 2010b. Norma Oficial Mexicana NOM-059-SEMARNAT-2010. Secretaría de Medio Ambiente y Recursos Naturales. Diario Oficial, 30 de diciembre de 2010. México, D.F. México. 78 pp.

Carbajal E., H. 2008. Importancia de las plantas en la cultura alimentaria de los xi'oi, Las Guapas, Rayón, S.L.P. Tesis de Maestría. Programa Multidisciplinario de Posgrado en Ciencias Ambientales, Universidad Autónoma de San Luis Potosí. San Luis Potosí, S.L.P., México. 136 pp.

Cartujano, S., S. Zamudio, O. Alcántara e I. Luna. 2002. El bosque mesófilo de montaña en el municipio de Landa de Matamoros, Querétaro, México. Bol. Soc. Bot. México 70: 13-43.

Foroughbakhch, R., V. Valdez, G. J. Alanís, M. C. Valadez y A. Rocha. 2004. Distribución relictual del bosque mesófilo de montaña (BMM) en el estado de Nuevo León. In: R. Foroughbakhch P., T. E. Torres C. y M. A. Alvarado V. (eds.). Tópicos selectos de botánica. Universidad Autónoma de Nuevo León. Monterrey, México. pp. 57-63.

García P., J. y J. Fortanelli M. 2005. Relicto de bosque de liquidámbar (Liquidambar styraciflua L.) en Tamasopo, San Luis Potosí, México. Simposio internacional "El conocimiento botánico en la gestión ambiental y el manejo de ecosistemas" y $2^{\circ}$ Simposio botánico del norte de México. Centro Interdisciplinario de Investigación para el Desarrollo Integral Regional Unidad Durango del Instituto Politécnico Nacional y Sociedad Botánica de México, A.C. Durango, México. p. 52.

García P., J. y J. Fortanelli M. 2010. Estudio florístico de dos relictos de bosque de niebla de San Luis Potosí. Resúmenes en CD del XVIII Congreso Mexicano de Botánica. Sociedad Botánica de México. Guadalajara, México, 21 al 27 de noviembre de 2010.

González E., M., J. A. Meave, F. G. Lorea H., G. Ibarra M., A. C. Newton (eds.). 2011. The red list of Mexican cloud forest trees. Fauna \& Flora International. Cambridge, UK. 148 pp.

Hernández X., E., H. Crum, W. B. Fox y A. J. Sharp. 1951. A unique vegetational area in Tamaulipas. Bull. Torrey Bot. Club 78(6): 458-463.

Leija L., E. G. 2010. Dinámica espacio-temporal del bosque mesófilo de montaña en la región fisiográfica Sierra Madre Oriental de San Luis Potosí, México. Tesis de licenciatura en Geografía. Coordinación de Ciencias Sociales y Humanidades, Universidad Autónoma de San Luis Potosí. San Luis Potosí, S.L.P., México. 104 pp.

Leija L., E. G., H. Reyes H., J. Fortanelli M. y G. Palacio A. 2011. Situación actual del bosque de niebla en el estado de San Luis Potosí, México. Investigación y Ciencia 53: 3-11

Luna, I., S. Ocegueda y O. Alcántara. 1994. Florística y notas biogeográficas del bosque mesófilo de montaña del municipio de Tlanchinol, Hidalgo, México. An. Inst. Biol. Univ. Nac. Aut. Méx. Ser. Bot. 65: 31-62.

Martin, P. S. 1955. Zonal distribution of vertebrates in a Mexican cloud forest. Amer. Nat. 89(849): 347-361. 
Miranda, F. 1947. Estudios sobre la vegetación de México. V. Rasgos de la vegetación en la Cuenca del Río de Las Balsas. Rev. Soc. Mex. Hist. Nat. 8: 95-114.

Miranda, F. y A. J. Sharp. 1950. Characteristics of the vegetation in certain temperate regions of Eastern Mexico. Ecology 31(3): 313-333.

Moreno, N. P. 1984. Glosario botánico ilustrado. Instituto Nacional de Investigaciones sobre Recursos Bióticos. Xalapa, México. 300 pp.

Mueller-Dombois, D. y H. Ellenberg. 1974. Aims and methods of vegetation ecology. John Wiley and Sons. New York, USA. 547 pp.

Puig, H. 1991. Vegetación de la Huasteca México. Estudio fitogeográfico y ecológico. Institut Français de Recherche Scientifique pour le Développement en Coopération (ORSTOM); Instituto de Ecología, A.C.; Centre d'Etudes Mexicaines et Centraméricaines (CEMCA). México, D.F., México. 625 pp.

Puig, H., R. Bracho y V. J. Sosa. 1987. El bosque mesófilo de montaña: composición florística y estructura. In: Puig, H. y R. Bracho (eds.). El bosque mesófilo de montaña de Tamaulipas. Instituto de Ecología, A.C. México, D.F., México. pp. 55-79.

Rzedowski, J. 1965. Vegetación del estado de San Luis Potosí. Acta Científica Potosina 1-2: 5-291.

Rzedowski, J. 1978. Vegetación de México. Limusa. México, D.F., México. 432 pp.

Smith, T. M. y R. L. Smith. 2002. Ecología. Pearson Educación. Madrid, España. 776 pp.

Stiling, P. 1999. Ecology; Theories and aplications. 3a. ed. Prentice Hall. New Jersey, USA. 840 pp.

Villaseñor, J. L. 2010. El bosque húmedo de montaña de México y sus plantas vasculares: catálogo florístico-taxonómico. Comisión Nacional para el Conocimiento y Uso de la Biodiversidad; Universidad Nacional Autónoma de México. México, D.F., México. $40 \mathrm{pp}$.

Williams L., G. 2007. El bosque de niebla del centro de Veracruz. Comisión Nacional para el Conocimiento y Uso de la Biodiversidad; Instituto de Ecología A.C. Xalapa, México. 204 pp. 


\section{APÉNDICE 1}

Lista florística del bosque de niebla de Copalillos, S.L.P. A = árbol; $\mathrm{a}=$ arbusto; $\mathrm{H}=$ hierba; $\mathrm{Tl}=$ trepadora leñosa; $\mathrm{Th}=$ trepadora no leñosa; $\mathrm{E}=$ epífita; $\mathrm{P}=$ parásita; $\mathrm{HP}=$ hemiparásita.

\begin{tabular}{|c|c|c|c|}
\hline Nombre científico & $\begin{array}{c}\text { Forma } \\
\text { vital }\end{array}$ & Nombre científico & $\begin{array}{c}\text { Forma } \\
\text { vital }\end{array}$ \\
\hline Acanthaceae & & Ilex decidua Walter & $\mathrm{a}$ \\
\hline $\begin{array}{l}\text { Justicia brandegeeana Wassh. \& L. B. } \\
\text { Sm. }\end{array}$ & $\mathrm{H}$ & $\begin{array}{l}\text { Ilex rubra } \mathrm{S} \text {. Watson } \\
\text { Araceae }\end{array}$ & A \\
\hline $\begin{array}{l}\text { Pseuderanthemum praecox (Benth.) } \\
\text { Leonard }\end{array}$ & $\mathrm{H}$ & Arisaema macrospathum Benth. & $\mathrm{H}$ \\
\hline Actinidiaceae & & Syngonium podophyllum Schott & Th \\
\hline $\begin{array}{l}\text { Saurauia aspera Turcz. } \\
\text { Adoxaceae }\end{array}$ & a & $\begin{array}{l}\text { Xanthosoma robustum Schott } \\
\text { Araliaceae }\end{array}$ & $\mathrm{H}$ \\
\hline $\begin{array}{l}\text { Sambucus canadensis L. } \\
\text { Alstroemeriaceae }\end{array}$ & A & $\begin{array}{l}\text { Dendropanax arboreus (L.) Decne. \& } \\
\text { Planch. }\end{array}$ & A \\
\hline $\begin{array}{l}\text { Bomarea hirtella (Kunth) Herb. } \\
\text { Altingiaceae }\end{array}$ & Th & $\begin{array}{l}\text { Aspleniaceae } \\
\text { Asplenium monanthes L. } \\
\text { Asteraceae }\end{array}$ & $\mathrm{H}$ \\
\hline $\begin{array}{l}\text { Liquidambar styraciflua } \mathrm{L} . \\
\text { Amaranthaceae }\end{array}$ & A & Acmella repens (Walter) Rich. & $\mathrm{H}$ \\
\hline Iresine diffusa Humb. \& Bonpl. ex Willd. & $\mathrm{H}$ & $\begin{array}{l}\text { Ageratina petiolaris (Moc. \& Sessé ex } \\
\text { DC.) R. M. King \& H. Rob. }\end{array}$ & a \\
\hline Anacardiaceae & & Calea ternifolia Kunth & $\mathrm{a}$ \\
\hline $\begin{array}{l}\text { Rhus aromatica var. trilobata (Nutt.) A. } \\
\text { Gray ex S. Watson }\end{array}$ & $\mathrm{a}$ & Calea urticifolia (Mill.) DC. & $\mathrm{H}$ \\
\hline Toxicodendron radicans (L.) Kuntze & $\mathrm{Tl}$ & $\begin{array}{l}\text { Chaptalia nutans (L.) Pol. } \\
\text { Cirsium mexicanum DC. }\end{array}$ & $\mathrm{H}$ \\
\hline Echeandia mexicana Cruden & $\mathrm{H}$ & $\begin{array}{l}\text { Elephantopus mollis Kunth } \\
\text { Erigeron longipes DC. }\end{array}$ & $\mathrm{H}$ \\
\hline $\begin{array}{l}\text { Apiaceae } \\
\text { Cyclospermum leptophyllum (Pers.) }\end{array}$ & $\mathrm{H}$ & $\begin{array}{l}\text { Fleischmannia pycnocephala (Less.) R. } \\
\text { M. King \& H. Rob. }\end{array}$ & $\mathrm{H}$ \\
\hline $\begin{array}{l}\text { Sprague ex Britton \& P.Wilson } \\
\text { Apocynaceae }\end{array}$ & & $\begin{array}{l}\text { Hymenostephium cordatum (Hook. \& } \\
\text { Arn.) S. F. Blake }\end{array}$ & $\mathrm{H}$ \\
\hline $\begin{array}{l}\text { Asclepias curassavica L. } \\
\text { Gonolobus niger (Cav.) R. Br. ex Schult. }\end{array}$ & $\begin{array}{l}\mathrm{H} \\
\mathrm{Tl}\end{array}$ & $\begin{array}{l}\text { Perymenium ovalifolium (A. Gray) B. L. } \\
\text { Turner }\end{array}$ & $\mathrm{Tl}$ \\
\hline Marsdenia pringlei $\mathrm{S}$. Watson & Th & $\begin{array}{l}\text { Roldana aschenborniana (Schauer) H. } \\
\text { Rob. \& Brettell }\end{array}$ & a \\
\hline $\begin{array}{l}\text { Metastelma angustifolium Turcz. } \\
\text { Aquifoliaceae }\end{array}$ & Th & $\begin{array}{l}\text { Roldana lanicaulis (Greenm.) H. Rob. \& } \\
\text { Brettell }\end{array}$ & $\mathrm{a}$ \\
\hline Ilex condensata Turcz. & A & Rumfordia alcortae Rzed. & $\mathrm{H}$ \\
\hline
\end{tabular}


Apéndice 1. Continuación.

\begin{tabular}{|c|c|c|c|}
\hline Nombre científico & $\begin{array}{c}\text { Forma } \\
\text { vital }\end{array}$ & Nombre científico & $\begin{array}{c}\text { Forma } \\
\text { vital }\end{array}$ \\
\hline Salmea scandens (L.) DC. & $\mathrm{Tl}$ & Clethraceae & \\
\hline Schistocarpha bicolor Less. & $\mathrm{H}$ & Clethra pringlei $\mathrm{S}$. Watson & A \\
\hline $\begin{array}{l}\text { Sclerocarpus uniserialis (Hook.) Benth. } \\
\text { \& Hook f. }\end{array}$ & $\mathrm{H}$ & Convolvulaceae & \\
\hline Stevia monardifolia Kunth & $\mathrm{H}$ & $\begin{array}{l}\text { Ipomoea purpurea }(\mathrm{L} .) \text { Roth } \\
\text { Cornaceae }\end{array}$ & Th \\
\hline Tagetes filifolia Lag. & $\mathrm{H}$ & Cornus excelsa Kunth & $\mathrm{a}$ \\
\hline Tagetes lucida Cav. & $\mathrm{H}$ & Crassulaceae & \\
\hline Vernonia liatroides DC. & $\mathrm{a}$ & Echeveria rosea Lindl. & $\mathrm{E}$ \\
\hline Vernonia patens Kunth & $\mathrm{a}$ & Cyatheaceae & \\
\hline $\begin{array}{l}\text { Begoniaceae } \\
\text { Begonia incarnata Link \& Otto }\end{array}$ & $\mathrm{H}$ & $\begin{array}{l}\text { Alsophila firma (Baker) D.S. Conant } \\
\text { Cyperaceae }\end{array}$ & A \\
\hline $\begin{array}{l}\text { Berberidaceae } \\
\text { Berberis gracilis Hartw. ex Benth. }\end{array}$ & $\mathrm{a}$ & $\begin{array}{l}\text { Cyperus hermaphroditus (Schltdl. \& } \\
\text { Cham.) Standl. }\end{array}$ & $\mathrm{H}$ \\
\hline $\begin{array}{l}\text { Betulaceae } \\
\text { Ostrya virginiana (Mill.) K. Koch } \\
\text { Bignoniaceae }\end{array}$ & A & $\begin{array}{l}\text { Rhynchospora radicans (Schltdl. \& } \\
\text { Cham.) H. Pfeiff. } \\
\text { Dennstaedtiaceae }\end{array}$ & $\mathrm{H}$ \\
\hline $\begin{array}{l}\text { (Schltdl. \& Cham.) Standl. } \\
\text { Amphilochium } \\
\text { (Signolle }\end{array}$ & $\mathrm{Tl}$ & $\begin{array}{l}\text { Dennstaedtia distenta (Kuntze) T. } \\
\text { Moore } \\
\text { Ebenaceae }\end{array}$ & $\mathrm{a}$ \\
\hline $\begin{array}{l}\text { Blechnaceae } \\
\text { Blechnum appendiculatum Willd. } \\
\text { Bromeliaceae }\end{array}$ & $\mathrm{H}$ & $\begin{array}{l}\text { Diospyros riojae Gómez Pompa } \\
\text { Euphorbiaceae }\end{array}$ & A \\
\hline $\begin{array}{l}\text { Bromellaceae } \\
\text { Tillandsia bartramii Elliott }\end{array}$ & $\mathrm{E}$ & $\begin{array}{l}\text { Bernardia dodecandra (Sessé ex Cav.) } \\
\text { McVaugh }\end{array}$ & $\mathrm{a}$ \\
\hline $\begin{array}{l}\text { Tillandsia deppeana Steud. } \\
\text { Cactaceae }\end{array}$ & E & $\begin{array}{l}\text { Cnidoscolus multilobus (Pax) I. M. } \\
\text { Johnst. }\end{array}$ & A \\
\hline $\begin{array}{l}\text { Selenicereus spinulosus (DC.) Britton \& } \\
\text { Rose }\end{array}$ & Th & $\begin{array}{l}\text { Croton cortesianus Kunth } \\
\text { Fabaceae }\end{array}$ & $\mathrm{a}$ \\
\hline Campanulaceae & & Amicia zygomeris DC. & $\mathrm{H}$ \\
\hline Lobelia sartorii Vatke & $\mathrm{H}$ & Amphicarpaea bracteata $($ L.) Fernald & Th \\
\hline Cannabaceae & & Bauhinia chapulhuacania Wunderlin & A \\
\hline Trema micrantha (L.) Blume & A & Canavalia villosa Benth. & Th \\
\hline Caprifoliaceae & & Centrosema virginianum (L.) Benth. & Th \\
\hline Lonicera pilosa (Kunth) Willd. & $\mathrm{Tl}$ & Cercis canadensis $\mathrm{L}$. & A \\
\hline Celastraceae & & Cologania broussonetii (Balb.) DC. & $\mathrm{H}$ \\
\hline Wimmeria concolor Schltdl. \& Cham. & A & Desmodium caripense (Kunth) G. Don & $\mathrm{H}$ \\
\hline
\end{tabular}


Apéndice 1. Continuación.

\begin{tabular}{|c|c|c|c|}
\hline Nombre científico & $\begin{array}{l}\text { Forma } \\
\text { vital }\end{array}$ & Nombre científico & $\begin{array}{c}\text { Forma } \\
\text { vital }\end{array}$ \\
\hline $\begin{array}{l}\text { Desmodium glutinosum (Muhl. ex Willd.) } \\
\text { Alph. Wood }\end{array}$ & $\mathrm{Th}$ & $\begin{array}{l}\text { Juglans mollis Engelm. } \\
\text { Lamiaceae }\end{array}$ & $\mathrm{A}$ \\
\hline Desmodium prehensile Schltdl. & $\mathrm{H}$ & Micromeria brownei (Sw.) Benth. & $\mathrm{H}$ \\
\hline $\begin{array}{l}\text { Macroptilium gibbosifolium (Ortega) A. } \\
\text { Delgado }\end{array}$ & $\mathrm{H}$ & Ocimum selloi Benth. & $\mathrm{H}$ \\
\hline Phaseolus coccineus $\mathrm{L}$. & Th & Salvia mexicana $\mathrm{L}$ & a \\
\hline $\begin{array}{l}\text { Senna septemtrionalis (Viv.) H.S. Irwin \& } \\
\text { Barneby }\end{array}$ & a & $\begin{array}{l}\text { Teucrium cubense Jacq. ssp. cubense } \\
\text { Lauraceae }\end{array}$ & $\mathrm{H}$ \\
\hline $\begin{array}{l}\text { Vigna adenantha (G. Mey.) Maréchal, } \\
\text { Mascherpa \& Stainier }\end{array}$ & Th & $\begin{array}{l}\text { Cinnamomum effusum (Meisn.) Kosterm. } \\
\text { Persea americana Mill. }\end{array}$ & $\begin{array}{l}\text { A } \\
\text { A }\end{array}$ \\
\hline $\begin{array}{l}\text { Zapoteca portoricensis (Jacq.) H. M. } \\
\text { Hern. }\end{array}$ & a & Persea liebmannii $\mathrm{Mez}$ & A \\
\hline Zornia thymifolia Kunth & $\mathrm{H}$ & Loganiaceae & \\
\hline Fagaceae & & Gelsemium sempervirens (L.) J. St.-Hil & $\mathrm{Tl}$ \\
\hline Quercus affinis Scheidw. & A & Lophosoriaceae & \\
\hline Quercus germana Schltdl. \& Cham. & A & $\begin{array}{l}\text { Lophosoria quadripinnata (J. F. Gmel.) } \\
\text { C. Chr. }\end{array}$ & $\mathrm{H}$ \\
\hline Quercus laeta Liebm. & A & Loranthaceae & \\
\hline Quercus obtusata Humb. \& Bonpl. & A & Phoradendron sp. & HP \\
\hline Quercus pinnativenulosa C. H. Mull. & A & Lythraceae & \\
\hline Quercus polymorpha Schltdl. \& Cham. & A & Heimia salicifolia (Kunth) Link & A \\
\hline Quercus rysophylla Weath. & A & Magnoliaceae & \\
\hline Quercus xalapensis Liebm. & A & Magnolia schiedeana Schltdl. & A \\
\hline Gesneriaceae & & Malpighiaceae & \\
\hline $\begin{array}{l}\text { Achimenes grandiflora (Schiede) DC. } \\
\text { Heliconiaceae }\end{array}$ & $\mathrm{H}$ & $\begin{array}{l}\text { Heteropterys beecheyana A. Juss. } \\
\text { Malvaceae }\end{array}$ & $\mathrm{Tl}$ \\
\hline Heliconia schiedeana Klotzsch & $\mathrm{H}$ & Guazuma ulmifolia Lam. & A \\
\hline Hypericaceae & & Pavonia schiedeana Steud. & $\mathrm{H}$ \\
\hline Ascyrum hypericoides L. & $\mathrm{H}$ & Pavonia uniflora (Sessé \& Moc.) Fryxell & $\mathrm{H}$ \\
\hline Hypoxidaceae & & Sida acuta Burm. f. & $\mathrm{H}$ \\
\hline $\begin{array}{l}\text { Hypoxis mexicana Schult. \& Schult. f. } \\
\text { Iridaceae }\end{array}$ & $\mathrm{H}$ & $\begin{array}{l}\text { Triumfetta semitriloba Jacq. } \\
\text { Marattiaceae }\end{array}$ & A \\
\hline $\begin{array}{l}\text { Tigridia pavonia (L. f.) DC. } \\
\text { Juglandaceae }\end{array}$ & $\mathrm{H}$ & $\begin{array}{l}\text { Marattia weinmanniifolia Liebm. } \\
\text { Melastomataceae }\end{array}$ & A \\
\hline $\begin{array}{l}\text { Carya ovata var. mexicana (Engelm. ex } \\
\text { Hemsl.) W. E. Manning }\end{array}$ & A & $\begin{array}{l}\text { Leandra cornoides (Schltdl. \& Cham.) } \\
\text { Cogn. }\end{array}$ & A \\
\hline
\end{tabular}


Apéndice 1. Continuación.

\begin{tabular}{|c|c|c|c|}
\hline Nombre científico & $\begin{array}{c}\text { Forma } \\
\text { vital }\end{array}$ & Nombre científico & $\begin{array}{c}\text { Forma } \\
\text { vital }\end{array}$ \\
\hline Tibouchina galeottiana (Naudin) Cogn. & $\mathrm{H}$ & Piperaceae & \\
\hline Meliaceae & & Peperomia blanda (Jacq.) Kunth & $\mathrm{H}$ \\
\hline Trichilia havanensis Jacq. & A & Piper auritum Kunth & A \\
\hline Menispermaceae & & Piper umbellatum L. & A \\
\hline Cissampelos pareira $\mathrm{L}$. & Th & Plantaginaceae & \\
\hline Moraceae & & Plantago australis ssp. hirtella (Kunth) & $\mathrm{H}$ \\
\hline Morus celtidifolia Kunth & A & $\begin{array}{l}\text { Rahn } \\
\text { Plantago lanceolata } \mathrm{L} \text {. }\end{array}$ & $\mathrm{H}$ \\
\hline $\begin{array}{l}\text { Myrsine ssp. coriacea (Sw.) R. Br. ex } \\
\text { Roem. \& Schult. }\end{array}$ & A & $\begin{array}{l}\text { Plantago nivea Kunth } \\
\text { Poaceae }\end{array}$ & $\mathrm{H}$ \\
\hline Myrtaceae & & Dichanthelium laxiflorum (Lam.) Gould & $\mathrm{H}$ \\
\hline $\begin{array}{l}\text { Eugenia xalapensis (Kunth) DC. } \\
\text { Psidium guaiava L. }\end{array}$ & A & $\begin{array}{l}\text { Pennisetum clandestinum Hochst. ex } \\
\text { Chiov. }\end{array}$ & $\mathrm{H}$ \\
\hline Onagraceae & & Setaria parviflora (Poir.) Kerguélen & $\mathrm{H}$ \\
\hline $\begin{array}{l}\text { Oenothera rosea L'Her. ex Aiton } \\
\text { Ophioglossaceae }\end{array}$ & $\mathrm{H}$ & $\begin{array}{l}\text { Sporobolus indicus (L.) R. Br. } \\
\text { Polypodiaceae }\end{array}$ & $\mathrm{H}$ \\
\hline Botrychium schaffneri Underw. & $\mathrm{H}$ & Campyloneurum angustifolium (Sw.) Fée & $\mathrm{E}$ \\
\hline Orchidaceae & & $\begin{array}{l}\text { Pecluma plumula (Humb. \& Bonpl. ex } \\
\text { Willd.) M. G. Price }\end{array}$ & $\mathrm{H}$ \\
\hline $\begin{array}{l}\text { Aulosepalum ramentaceum (Lindl.) } \\
\text { Garay }\end{array}$ & $\mathrm{H}$ & Polypodium colpodes Kunze & $\mathrm{H}$ \\
\hline $\begin{array}{l}\text { Cyclopogon comosus (Rchb. f.) Burns- } \\
\text { Bal. }\end{array}$ & $\mathrm{H}$ & $\begin{array}{l}\text { Polypodium furfuraceum Schltdl. \& } \\
\text { Cham. }\end{array}$ & $\mathrm{H}$ \\
\hline $\begin{array}{l}\text { Cyclopogon violaceus (A. Rich. \& } \\
\text { Galeotti) Schltr. }\end{array}$ & $\mathrm{H}$ & $\begin{array}{l}\text { Pteridaceae } \\
\text { Adiantum andicola Liebm. }\end{array}$ & $\mathrm{H}$ \\
\hline Isochilus unilateralis B. L. Rob. & $\mathrm{E}$ & Pteris orizabae M. Martens \& Galeotti & $\mathrm{H}$ \\
\hline Ponthieva racemosa (Walter) C. Mohr & $\mathrm{H}$ & Ranunculaceae & \\
\hline Orobanchaceae & & Clematis dioica $\mathrm{L}$. & $\mathrm{H}$ \\
\hline Castilleja arvensis Cham. \& Schltdl. & $\mathrm{H}$ & Rosaceae & \\
\hline $\begin{array}{l}\text { Conopholis alpina Liebm. } \\
\text { Passifloraceae }\end{array}$ & $\mathrm{P}$ & $\begin{array}{l}\text { Crataegus mexicana Moc. \& Sessé ex } \\
\text { DC. }\end{array}$ & A \\
\hline Passiflora coriacea Juss. & Th & Prunus persica $(\mathrm{L}$.$) Batsch$ & A \\
\hline Phytolaccaceae & & Prunus serotina Ehrh. & A \\
\hline Phytolacca icosandra L. & $\mathrm{H}$ & Rubus adenotrichos Schltdl. & A \\
\hline $\begin{array}{l}\text { Phytolacca rivinoides Kunth \& C. D. } \\
\text { Bouché }\end{array}$ & $\mathrm{H}$ & $\begin{array}{l}\text { Rubiaceae } \\
\text { Bouvardia laevis M. Martens \& Galeotti }\end{array}$ & A \\
\hline
\end{tabular}


Apéndice 1. Continuación.

\begin{tabular}{|c|c|c|c|}
\hline Nombre científico & $\begin{array}{l}\text { Forma } \\
\text { vital }\end{array}$ & Nombre científico & $\begin{array}{c}\text { Forma } \\
\text { vital }\end{array}$ \\
\hline Chione venosa subsp. mexicana (Standl.) & $\mathrm{A}$ & Physalis volubilis Waterf. & $\mathrm{H}$ \\
\hline & & Solanum myriacanthum Dunal & A \\
\hline Deppea purpusii Standl. & A & Solanum seaforthianum Andrews & $\mathrm{Tl}$ \\
\hline $\begin{array}{l}\text { Galium hypocarpium (L.) Endl. ex } \\
\text { Griseb. }\end{array}$ & $\mathrm{H}$ & Solanum torvum $\mathrm{Sw}$. & A \\
\hline Galium mexicanum Kunth & $\mathrm{H}$ & Styracaceae & \\
\hline Hamelia patens Jacq. & A & Styrax glabrescens Benth. & A \\
\hline Hoffmannia aff. excelsa (Kunth.) K. & A & Tectariaceae & \\
\hline Schum. & & Ctenitis melanosticta (Kunze) Copel. & $\mathrm{H}$ \\
\hline Hoffmannia montana L. O. Williams & $\mathrm{H}$ & Thymelaeaceae & \\
\hline Randia laetevirens Standl. & A & Daphnopsis mollis (Meisn.) Standl. & A \\
\hline Rutaceae & & Urticaceae & \\
\hline Zanthoxylum clava-herculis L. & A & Pilea microphylla (L.) Liebm. & $\mathrm{H}$ \\
\hline Salicaceae & & Pilea pubescens Liebm. & $\mathrm{H}$ \\
\hline Salix taxifolia Kunth & A & Urera caracasana (Jacq.) Griseb. & $\mathrm{H}$ \\
\hline Xylosma flexuosa (Kunth) Hemsl. & A & Verbenaceae & \\
\hline Sapindaceae & & Glandularia elegans (Kunth) Umber & $\mathrm{H}$ \\
\hline Sapindus saponaria L. & A & Violaceae & \\
\hline Sapotaceae & & Viola hookeriana Kunth & $\mathrm{H}$ \\
\hline Sideroxylon palmeri (Rose) T. D. Penn. & A & Vitaceae & \\
\hline Selaginellaceae & & Vitis berlandieri Planch. & $\mathrm{Tl}$ \\
\hline Selaginella stenophylla A. Braun & $\mathrm{H}$ & Vittariaceae & \\
\hline Smilacaceae & & Vittaria graminifolia Kaulf. & $\mathrm{H}$ \\
\hline Smilax bona-nox L. & Th & Woodsiaceae & \\
\hline Smilax glauca Walter & $\mathrm{Tl}$ & Diplazium franconis Liebm. & $\mathrm{H}$ \\
\hline Smilax mollis Humb. \& Bonpl. ex Willd & Th & Zamiaceae & \\
\hline Smilax moranensis M. Martens \& Galeotti & Th & Ceratozamia mexicana var. latifolia & A \\
\hline Smilax pringlei Greenm. & Th & (Miq.) J. Schust. & \\
\hline Solanaceae & & Zingiberaceae & \\
\hline Brugmansia candida Pers. & A & Zingiber officinale Roscoe & $\mathrm{H}$ \\
\hline Cestrum oblongifolium Schltdl. & A & & \\
\hline
\end{tabular}




\section{APÉNDICE 2}

Especies del estrato arbóreo de Copalillos, San Luis Potosí, y de bosques situados hacia el sur (Landa de Matamoros, Querétaro y Tlanchinol, Hidalgo) y hacia el norte (El Cielo, Tamaulipas).

\begin{tabular}{|c|c|c|c|c|}
\hline Nombre científico & $\begin{array}{c}\text { Tlanchinol } \\
\text { Luna et al., } \\
1994\end{array}$ & $\begin{array}{c}\text { Landa* } \\
\text { Cartujano et } \\
\text { al., } 2002\end{array}$ & Copalillos & $\begin{array}{c}\text { El Cielo } \\
\text { Puig, et al., } \\
1987\end{array}$ \\
\hline Acacia angustissima (Mill.) Kuntze & & & & $\mathrm{X}$ \\
\hline $\begin{array}{l}\text { Acer saccharum ssp. skutchii (Rehder) A. } \\
\text { E. Murray }\end{array}$ & & & & $\mathrm{X}$ \\
\hline Alchornea latifolia $\mathrm{Sw}$. & $\mathrm{X}$ & & & \\
\hline Alnus acuminata Kunth & $\mathrm{X}$ & & & \\
\hline Alsophila firma (Baker) D. S. Conant & $\mathrm{X}$ & & $\mathrm{X}$ & \\
\hline Aphananthe monoica (Hemsl.) J.-F. Leroy & & $\mathrm{X}$ & & \\
\hline Arctostaphylos sp. & $\mathrm{X}$ & & & \\
\hline Bauhinia chapulhuacania Wunderlin & & $\mathrm{X}$ & $\mathrm{X}$ & \\
\hline Befaria laevis Benth. & $\mathrm{X}$ & & & \\
\hline Beilschmiedia mexicana $(\mathrm{Mez})$ Kosterm & $\mathrm{X}$ & $\mathrm{X}$ & & \\
\hline Berberis gracilis Lindl. & & & & $\mathrm{X}$ \\
\hline $\begin{array}{l}\text { Bernardia dodecandra (Sessé ex Cav.) } \\
\text { Govaerts }\end{array}$ & & $\mathrm{X}$ & & $\mathrm{X}$ \\
\hline Boehmeria ulmifolia Wedd. & & $\mathrm{X}$ & & \\
\hline Buddleja cordata Kunth & $\mathrm{X}$ & & & \\
\hline Buddleja parviflora Kunth & $\mathrm{X}$ & & & \\
\hline Carpinus caroliniana Walter & $\mathrm{X}$ & & & \\
\hline $\begin{array}{l}\text { Carya ovata var. mexicana (Engelm. ex } \\
\text { Hemsl.) Manning }\end{array}$ & & $\mathrm{X}$ & $\mathrm{X}$ & $\mathrm{X}$ \\
\hline Cercis canadensis $\mathrm{L}$. & & $\mathrm{X}$ & $\mathrm{X}$ & $\mathrm{X}$ \\
\hline $\begin{array}{l}\text { Chione venosa var. mexicana (Standl.) } \\
\text { David W. Taylor }\end{array}$ & & & $\mathrm{X}$ & $\mathrm{X}$ \\
\hline $\begin{array}{l}\text { Cinnamomum bractifoliaceum Lorea- } \\
\text { Hern. }\end{array}$ & & $\mathrm{X}$ & & \\
\hline Cinnamoтит effusum (Meisn.) Kosterm. & & $\mathrm{X}$ & $\mathrm{X}$ & \\
\hline $\begin{array}{l}\text { Cinnamomum pachypodum (Nees) } \\
\text { Kosterm. }\end{array}$ & & $\mathrm{X}$ & & \\
\hline $\begin{array}{l}\text { Cinnamomum salicifolium (Nees) } \\
\text { Kosterm. }\end{array}$ & & $\mathrm{X}$ & & \\
\hline Clethra lanata M.Martens \& Galeotti & & $\mathrm{X}$ & & \\
\hline $\begin{array}{l}\text { Clethra macrophylla M.Martens \& } \\
\text { Galeotti }\end{array}$ & $\mathrm{X}$ & & & \\
\hline
\end{tabular}


Apéndice 2. Continuación.

\begin{tabular}{|c|c|c|c|c|}
\hline Nombre científico & $\begin{array}{c}\text { Tlanchinol } \\
\text { Luna et al., } \\
1994\end{array}$ & $\begin{array}{c}\text { Landa* } \\
\text { Cartujano et } \\
\text { al., } 2002\end{array}$ & Copalillos & $\begin{array}{c}\text { El Cielo } \\
\text { Puig, et al., } \\
1987\end{array}$ \\
\hline Clethra mexicana DC. & $\mathrm{X}$ & & & \\
\hline Clethra pringlei S. Watson & & $\mathrm{X}$ & $\mathrm{X}$ & $\mathrm{X}$ \\
\hline Cleyera theoides (Sw.) Choisy & & $\mathrm{X}$ & & \\
\hline $\begin{array}{l}\text { Cnidoscolus multilobus (Pax) I. M. } \\
\text { Johnst. }\end{array}$ & & & $\mathrm{X}$ & $\mathrm{X}$ \\
\hline Cornus disciflora Moc. \& Sessé ex DC. & $\mathrm{X}$ & $\mathrm{X}$ & & $\mathrm{X}$ \\
\hline Cornus excelsa Kunth & & $\mathrm{X}$ & & $\mathrm{X}$ \\
\hline $\begin{array}{l}\text { Crataegus mexicana Moc. \& Sessé ex } \\
\text { DC. }\end{array}$ & & & $\mathrm{X}$ & \\
\hline Cyathea aff. divergens Kunze & $\mathrm{X}$ & & & \\
\hline $\begin{array}{l}\text { Cyathea fulva (M. Martens \& Galeotti) } \\
\text { Fée }\end{array}$ & $\mathrm{X}$ & & & \\
\hline Dalbergia glomerata Hemsl. & $\mathrm{X}$ & & & \\
\hline Dalbergia palo-escrito Rzed. & & $\mathrm{X}$ & & \\
\hline Daphnopsis mollis (Meisn.) Standl. & & $\mathrm{X}$ & $\mathrm{X}$ & \\
\hline $\begin{array}{l}\text { Dendropanax arboreus (L.) Decne. \& } \\
\text { Planch. }\end{array}$ & & & $\mathrm{X}$ & $\mathrm{X}$ \\
\hline Diospyros riojae Gómez Pompa & & & $\mathrm{X}$ & $\mathrm{X}$ \\
\hline $\begin{array}{l}\text { Eugenia capuli (Schltdl. \& Cham.) Hook. } \\
\text { \& Arn. }\end{array}$ & & & & $\mathrm{X}$ \\
\hline Eugenia crenularis Lundell & & $\mathrm{X}$ & & \\
\hline Eugenia xalapensis (Kunth) DC. & $\mathrm{X}$ & $\mathrm{X}$ & $\mathrm{X}$ & \\
\hline Eugenia xilitlensis McVaugh & & $\mathrm{X}$ & & \\
\hline Forestiera reticulata Torr. & & $\mathrm{X}$ & & \\
\hline Garrya laurifolia Benth. & & $\mathrm{X}$ & & \\
\hline Gaultheria erecta Vent. & $\mathrm{X}$ & & & \\
\hline Guazuma ulmifolia Lam. & & & $\mathrm{X}$ & $\mathrm{X}$ \\
\hline Gymnanthes longipes Müll. Arg. & & $\mathrm{X}$ & & \\
\hline Gymnanthes lucida Sw. & $\mathrm{X}$ & & & \\
\hline Harpalyce arborescens A. Gray & & & & $X$ \\
\hline Heliocarpus americanus L. & & $\mathrm{X}$ & & \\
\hline Heliocarpus appendiculatus Turcz. & $\mathrm{X}$ & & & \\
\hline Ilex condensata Turcz. & & & $\mathrm{X}$ & \\
\hline Ilex decidua Walter & & $\mathrm{X}$ & & \\
\hline Ilex discolor Hemsl. & & $\mathrm{X}$ & & $\mathrm{X}$ \\
\hline $\begin{array}{l}\text { Ilex discolor var. tolucana (Hemsl.) } \\
\text { Edwin ex J. Linares }\end{array}$ & $\mathrm{X}$ & & & \\
\hline Ilex rubra $\mathrm{S}$. Watson & & & $\mathrm{X}$ & \\
\hline Illicium floridanum J. Ellis & $X$ & & & \\
\hline
\end{tabular}


Apéndice 2. Continuación.

\begin{tabular}{|c|c|c|c|c|}
\hline Nombre científico & $\begin{array}{c}\text { Tlanchinol } \\
\text { Luna et al., } \\
1994\end{array}$ & $\begin{array}{c}\text { Landa* } \\
\text { Cartujano et } \\
\text { al., } 2002\end{array}$ & Copalillos & $\begin{array}{c}\text { El Cielo } \\
\text { Puig, et al., } \\
1987\end{array}$ \\
\hline Inga huastecana M. Sousa & & $\mathrm{X}$ & & \\
\hline Inga latibracteata Harms & $\mathrm{X}$ & $\mathrm{X}$ & & \\
\hline Juglans mollis Engelm. & & $\mathrm{X}$ & $\mathrm{X}$ & $\mathrm{X}$ \\
\hline Juglans pyriformis Liebm. & $\mathrm{x}$ & & & \\
\hline Licaria campechiana (Standl.) Kosterm. & & $\mathrm{X}$ & & \\
\hline Liquidambar styraciflua $\mathrm{L}$. & $\mathrm{X}$ & $\mathrm{X}$ & $\mathrm{X}$ & $\mathrm{X}$ \\
\hline Litsea glaucescens Kunth & $\mathrm{X}$ & $\mathrm{X}$ & & \\
\hline Lonchocarpus rugosus Benth. & & $\mathrm{X}$ & & \\
\hline $\begin{array}{l}\text { Lyonia squamulosa M. Martens \& } \\
\quad \text { Galeotti }\end{array}$ & $\mathrm{X}$ & & & \\
\hline Lysiloma aff. microphylla Benth. & $\mathrm{X}$ & & & \\
\hline Magnolia schiedeana Schltl. & $\mathrm{x}$ & & $\mathrm{X}$ & $\mathrm{X}$ \\
\hline Meliosma alba (Schltdl.) Walp. & & & & $\mathrm{X}$ \\
\hline Meliosma oaxacana Standl. & & & & $\mathrm{X}$ \\
\hline Microtropis schiedeana Loes. & $\mathrm{X}$ & & & \\
\hline Morus celtidifolia Kunth & & & $\mathrm{X}$ & $\mathrm{X}$ \\
\hline Myrcianthes fragrans (Sw.) McVaugh & & $\mathrm{X}$ & & \\
\hline $\begin{array}{l}\text { Myrsine coriacea subsp. coriacea (Sw.) } \\
\text { R. Br. ex Roem. \& Schult. }\end{array}$ & $\mathrm{X}$ & $\mathrm{X}$ & $\mathrm{x}$ & $\mathrm{X}$ \\
\hline Nectandra salicifolia (Kunth) Nees & & $\mathrm{X}$ & & \\
\hline Nectandra sanguinea Rol. ex Rottb. & & $\mathrm{X}$ & & $\mathrm{X}$ \\
\hline Ocotea effusa (Meisn.) Hemsl. & $\mathrm{X}$ & & & \\
\hline Ocotea helicterifolia (Meisn.) Hemsl. & $\mathrm{X}$ & & & \\
\hline Ocotea klotzschiana (Nees) Hemsl. & & $\mathrm{X}$ & & \\
\hline Ocotea psychotrioides Billb. ex Meissner & & $\mathrm{X}$ & & \\
\hline $\begin{array}{l}\text { Oreopanax xalapensis (Kunth) Decne. \& } \\
\text { Planch. }\end{array}$ & $\mathrm{X}$ & & & \\
\hline Osmanthus americanus (L.) A. Gray & $\mathrm{X}$ & & & \\
\hline Ostrya virginiana (Mill.) K. Koch & $\mathrm{X}$ & $\mathrm{X}$ & $\mathrm{X}$ & $\mathrm{X}$ \\
\hline Perrottetia longistylis Rose & $\mathrm{X}$ & & & \\
\hline Perrottetia ovata Hemsl. & $\mathrm{X}$ & $\mathrm{X}$ & & \\
\hline Persea americana Mill. & & $\mathrm{X}$ & $\mathrm{X}$ & \\
\hline Persea liebmannii Mez & & $\mathrm{X}$ & $\mathrm{X}$ & \\
\hline Photinia mexicana (Baill.) Hemsl. & & $\mathrm{X}$ & & \\
\hline Picramnia xalapensis Planch. & $\mathrm{X}$ & $\mathrm{X}$ & & \\
\hline Pinus greggii Engelm. ex Parl. & $\mathrm{X}$ & & & \\
\hline Pinus patula Schiede ex Schltdl. \& Cham. & $\mathrm{X}$ & & & \\
\hline Pithecellobium insigne Micheli & & $\mathrm{X}$ & & \\
\hline
\end{tabular}


Apéndice 2. Continuación.

\begin{tabular}{|c|c|c|c|c|}
\hline Nombre científico & $\begin{array}{c}\text { Tlanchinol } \\
\text { Luna et al., } \\
1994\end{array}$ & $\begin{array}{c}\text { Landa* } \\
\text { Cartujano et } \\
\text { al., } 2002\end{array}$ & Copalillos & $\begin{array}{c}\text { El Cielo } \\
\text { Puig, et al., } \\
1987\end{array}$ \\
\hline Podocarpus matudae Lundell & $\mathrm{X}$ & & & $\mathrm{X}$ \\
\hline Prunus brachybotrya Zucc & $\mathrm{X}$ & & & \\
\hline Prunus serotina Ehrh. & & $\mathrm{X}$ & $\mathrm{X}$ & $\mathrm{X}$ \\
\hline Prunus sp. & & & & $\mathrm{X}$ \\
\hline Psidium guajava $\mathrm{L}$. & & & $\mathrm{X}$ & \\
\hline Quercus affinis Scheidw. & & $\mathrm{X}$ & $\mathrm{X}$ & \\
\hline Quercus eugeniifolia Liebm. & $\mathrm{X}$ & & & \\
\hline Quercus germana Schltdl. \& Cham. & $\mathrm{X}$ & $\mathrm{X}$ & $\mathrm{X}$ & $\mathrm{X}$ \\
\hline Quercus laeta Liebm. & & & $\mathrm{X}$ & \\
\hline Quercus obtusata Bonpl. & & & $\mathrm{X}$ & \\
\hline Quercus pinnativenulosa C. H. Mull. & & & $\mathrm{X}$ & \\
\hline Quercus polymorpha Schltdl. \& Cham. & & $\mathrm{X}$ & $\mathrm{X}$ & \\
\hline Quercus rysophylla Weath. & & $\mathrm{X}$ & $\mathrm{X}$ & \\
\hline Quercus sapotifolia Liebm. & $\mathrm{X}$ & & & \\
\hline Quercus xalapensis Bonpl. & $\mathrm{X}$ & $\mathrm{X}$ & $\mathrm{X}$ & $\mathrm{X}$ \\
\hline Rhamnus caroliniana Walter & & & & $\mathrm{X}$ \\
\hline Rhamnus longistyla C. B. Wolf & $\mathrm{X}$ & & & \\
\hline Rhamnus mucronata Schltdl. & $\mathrm{X}$ & & & \\
\hline $\begin{array}{l}\text { Rhamnus pompana M. C. Johnst. \& L. A. } \\
\text { Johnst. }\end{array}$ & $\mathrm{X}$ & & & \\
\hline $\begin{array}{l}\text { Robinsonella discolor Rose \& Baker f. } \\
\text { ex Rose }\end{array}$ & & $\mathrm{X}$ & & \\
\hline Robinsonella sp. & & & & $\mathrm{X}$ \\
\hline Sambucus canadensis L. & $\mathrm{X}$ & & $\mathrm{X}$ & \\
\hline Sapindus saponaria L. & & $\mathrm{X}$ & $\mathrm{X}$ & $\mathrm{X}$ \\
\hline Saurauia scabrida Hemsl. & $\mathrm{X}$ & $\mathrm{X}$ & & \\
\hline $\begin{array}{l}\text { Senna racemosa (Mill.) H. S. Irwin \& } \\
\text { Barneby }\end{array}$ & & $\mathrm{X}$ & & \\
\hline Sideroxylon palmeri (Rose) T. D. Penn. & & & $X$ & \\
\hline Sloanea sp. & $\mathrm{X}$ & & & \\
\hline Solanum aligerum Schltdl. & $\mathrm{X}$ & & & \\
\hline Styrax glabrescens Benth. & $\mathrm{X}$ & $\mathrm{X}$ & $\mathrm{X}$ & \\
\hline Symplocos speciosa Hemsl. & $\mathrm{X}$ & & & \\
\hline Taxus globosa Schltdl. & & & & $\mathrm{X}$ \\
\hline Ternstroemia sylvatica Schltdl. \& Cham. & & $\mathrm{X}$ & & $\mathrm{X}$ \\
\hline $\begin{array}{l}\text { Tilia americana var. mexicana (Schltdl.) } \\
\text { Hardin }\end{array}$ & & $\mathrm{X}$ & & $\mathrm{X}$ \\
\hline Trema micrantha $(\mathrm{L}$.$) Blume$ & $\mathrm{X}$ & & $\mathrm{X}$ & \\
\hline Trichilia havanensis Jacq. & $\mathrm{X}$ & & & \\
\hline
\end{tabular}


Apéndice 2. Continuación.

\begin{tabular}{|c|c|c|c|c|}
\hline Nombre científico & $\begin{array}{c}\text { Tlanchinol } \\
\text { Luna et al., } \\
1994\end{array}$ & $\begin{array}{l}\text { Landa* } \\
\text { Cartujano et } \\
\text { al., } 2002\end{array}$ & Copalillos & $\begin{array}{c}\text { El Cielo } \\
\text { Puig, et al. } \\
1987 \\
\end{array}$ \\
\hline Tridimeris hahniana Baill. & & $\mathrm{X}$ & & \\
\hline Turpinia occidentalis (Sw.) G. Don & $\mathrm{X}$ & $\mathrm{X}$ & & $\mathrm{X}$ \\
\hline Ulmus mexicana (Liebm.) Planch. & & $\mathrm{X}$ & & \\
\hline Vaccinium leucanthum Schltdl. & $\mathrm{X}$ & & & \\
\hline Vaccinium stenophyllum Steud. & $\mathrm{X}$ & & & \\
\hline Viburnum microcarpum Schltdl. \& Cham. & $\mathrm{X}$ & & & \\
\hline Viburnum tiliifolium (Oerst.) Hemsl. & $\mathrm{X}$ & & & \\
\hline Wimmeria concolor Schltdl. \& Cham. & & $\mathrm{X}$ & $\mathrm{X}$ & $\mathrm{X}$ \\
\hline Zanthoxylum acuminatum (Sw.) Sw. & & & & $\mathrm{X}$ \\
\hline Zanthoxylum clava-herculis L. & & $\mathrm{X}$ & $\mathrm{X}$ & \\
\hline $\begin{array}{l}\text { Zanthoxylum melanostictum Schltdl. \& } \\
\text { Cham. }\end{array}$ & $\mathrm{X}$ & & & \\
\hline
\end{tabular}

* Los datos que se presentan corresponden solo a la amplitud altitudinal de 800 a $1800 \mathrm{~m}$. 